\title{
Thermal Modeling of Zero Boil Off Tank Experiment
}

Erin M. Tesny, Daniel M. Hauser NASA Glenn Research Center

Presented By

Erin Tesny

ANALYSIS WORKSHOP

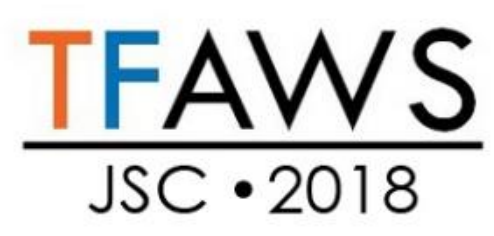

Thermal \& Fluids Analysis Workshop TFAWS 2018

August 20-24, 2018

NASA Johnson Space Center

Houston, TX 


\section{Outline}

- Background

- ZBOT Experiment Description

- Thermal Modeling \& Validation

- 1G Vacuum-Jacket Heating

- 1G Strip Heater

- Microgravity Strip Heater

- Conclusions \& Future Work 


\section{Background}

- Cryogenic Fluid Storage in microgravity is crucial to the development of future long-term space missions

- Zero Boil-Off Pressure Control:

- High cost savings

- Various design/implementation issues

- Two phase flow in microgravity, heat \& mass transfer interactions

- Creating accurate thermal models of cryogenic fluids is a key step in developing these systems 


\section{Zero Boil-Off Tank Experiment}

- Designed to investigate two-phase pressurization/depressurization in microgravity

- Working Fluid: Perfluoro-normal-Pentane (PNP)

- Experiment conducted on ISS, Fall 2017

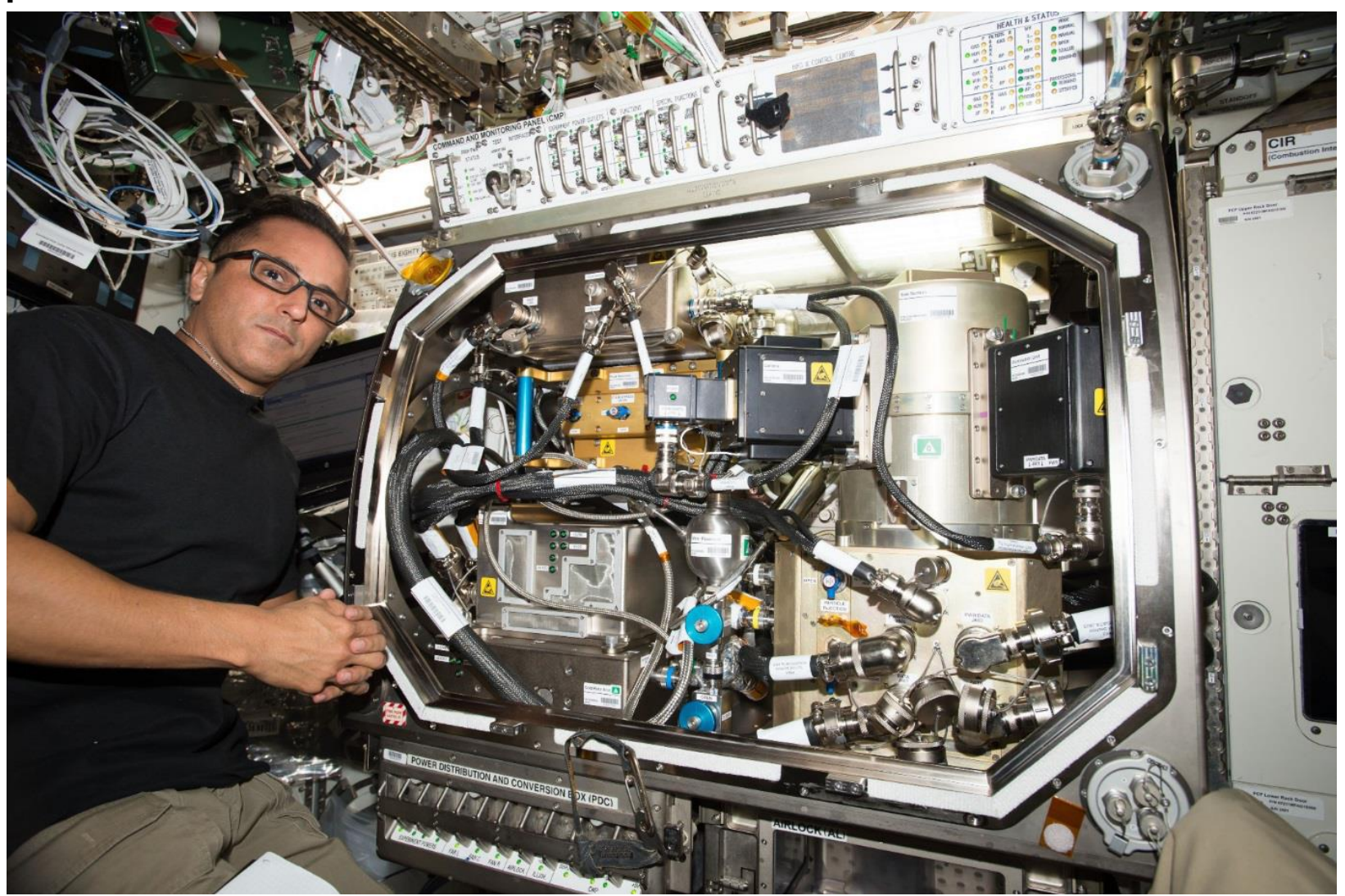




\section{ZBOT Test Setup}
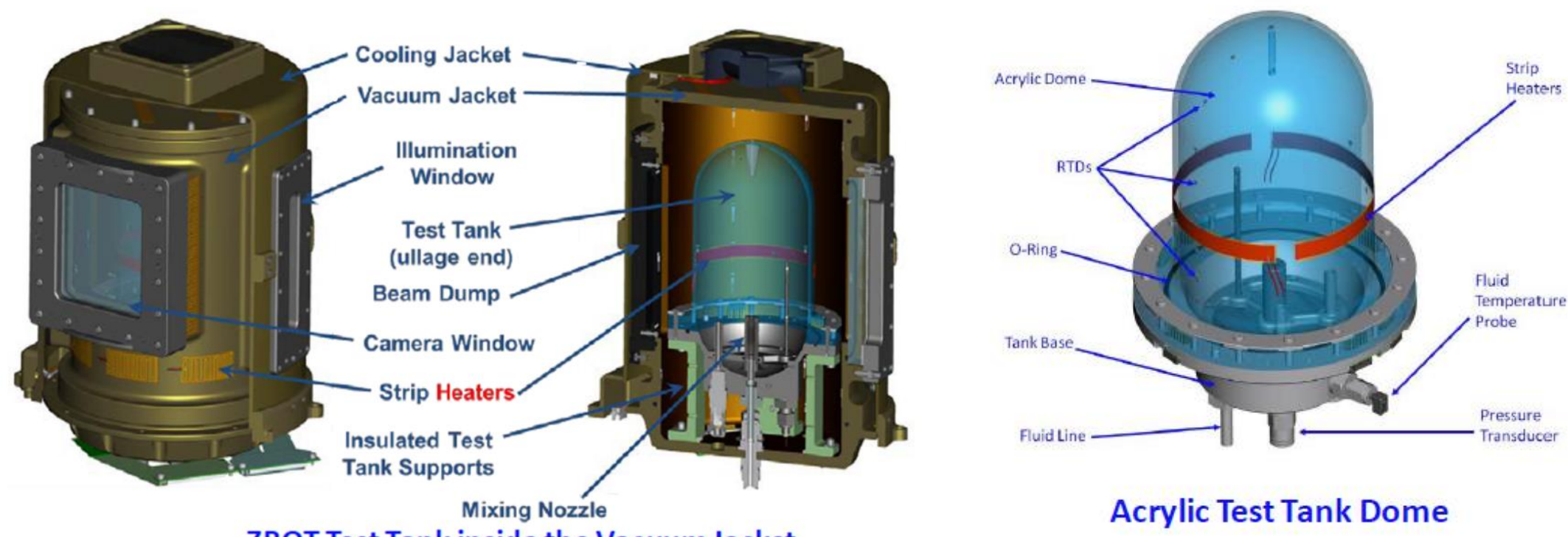

Acrylic Test Tank Dome

ZBOT Test Tank inside the Vacuum Jacket

- ZBOT-1

- Natural Convection

- Forced Mixing

- Microgravity Evolving Phase Distribution

- Free Surface Dynamics/Ullage Dynamics

- Evaporation/Condensation

- Superheating/Nucleate Boiling in Microgravity 


\section{Thermal Model}

- Geometry simplified in SpaceClaim

- Imported into Thermal Desktop

- Heat transfer from VJ to Tank Wall/Skirt via

- Radiation from VJ

- Conduction from VJ to Tank Wall/Skirt, through Air

- Conduction along Tank Wall, VJ wall

- Measured VJ temperatures from experiment used as Boundary Condition in model

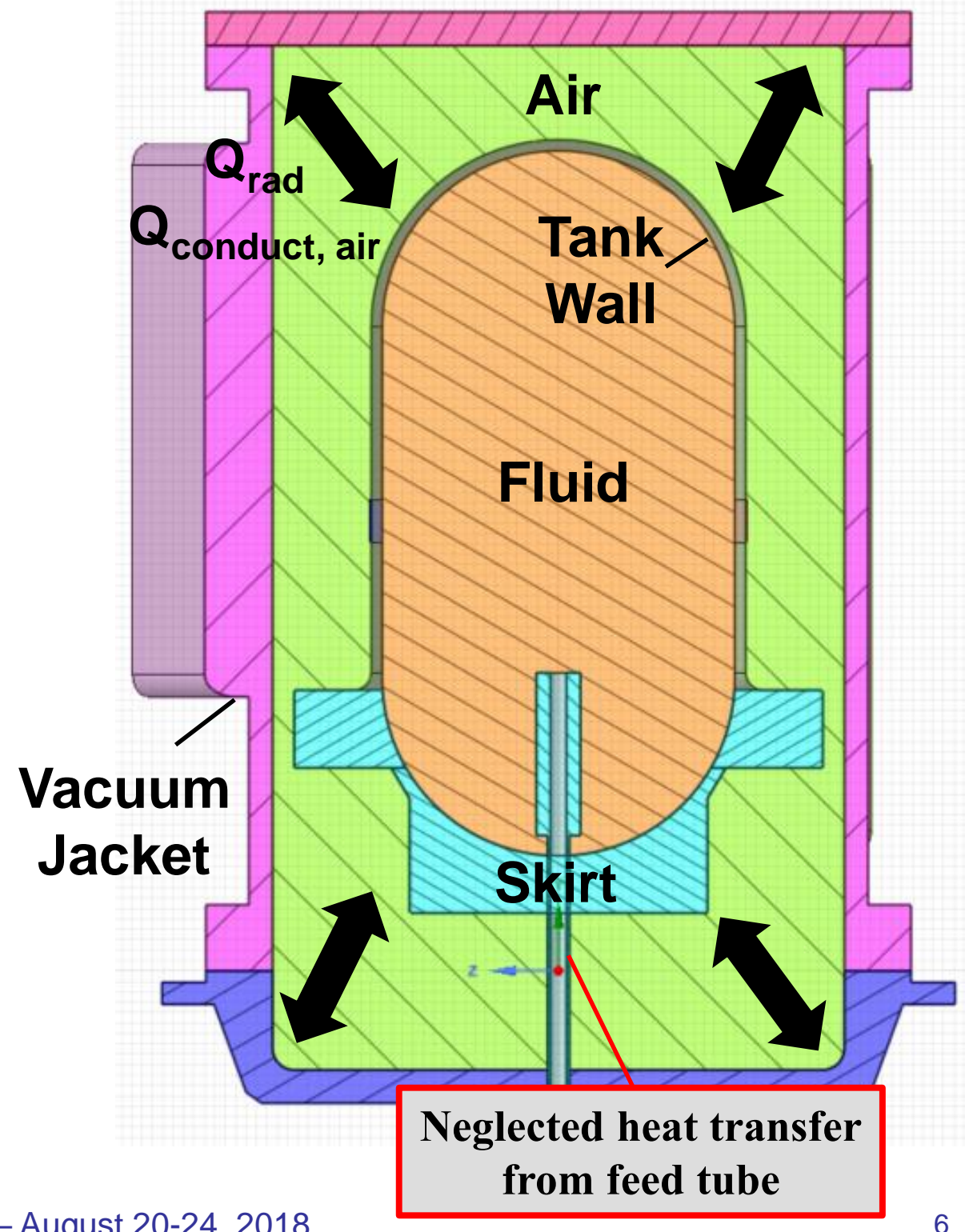


Ground Based Model Validation: 1G Self-Pressurization- VJ Heating

- Thermal Desktop and SINDA/FLUINT

- Vacuum Jacket Heating

$-\mathrm{Q}=0.5 \mathrm{~W}$

- Fill Level $=70 \%$

Pressure Change J1 Data \& Simulation

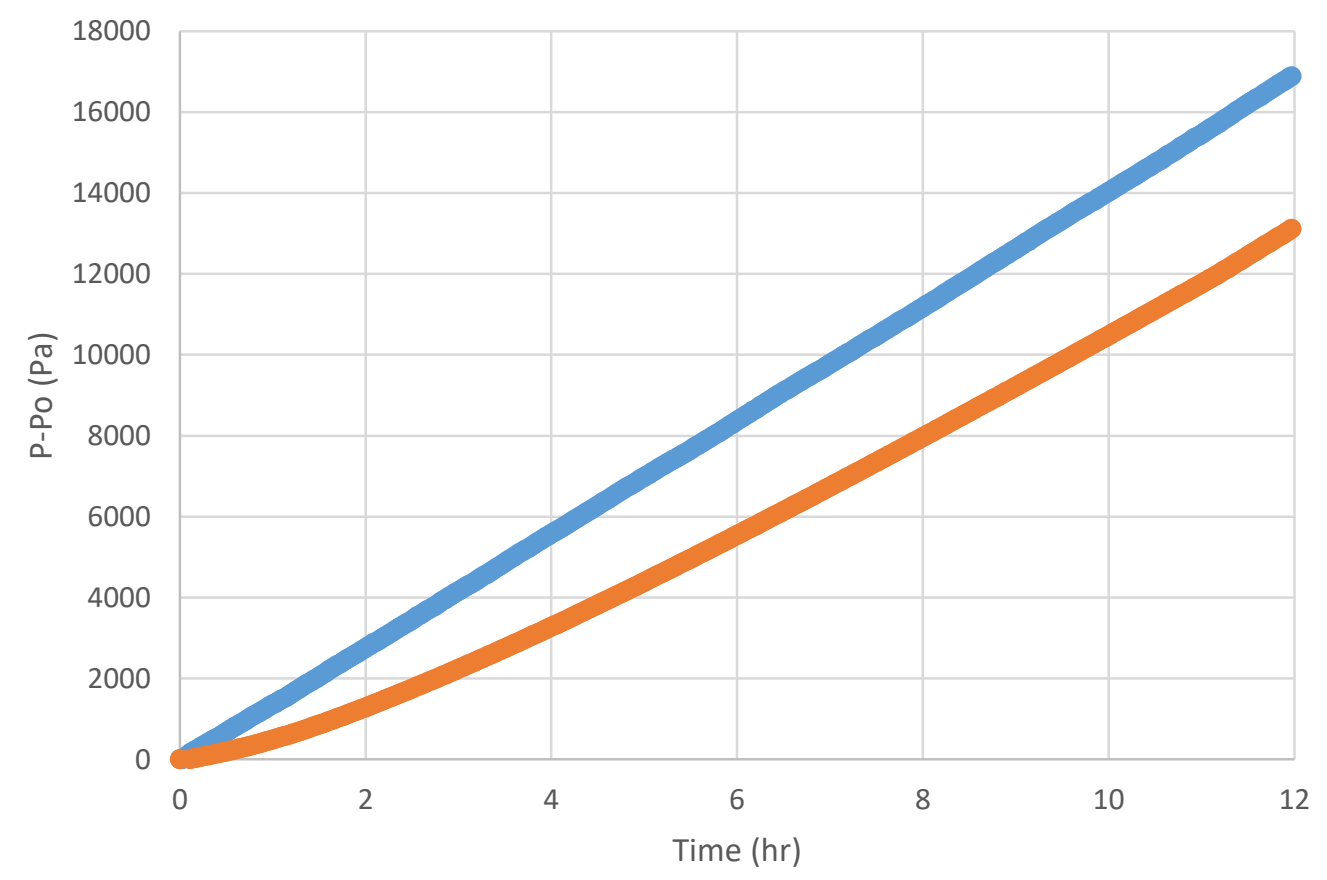

- Experimental Data TD Sim

TFAWS 2018 - August 20-24, 2018

Average Vacuum Jacket Temperature
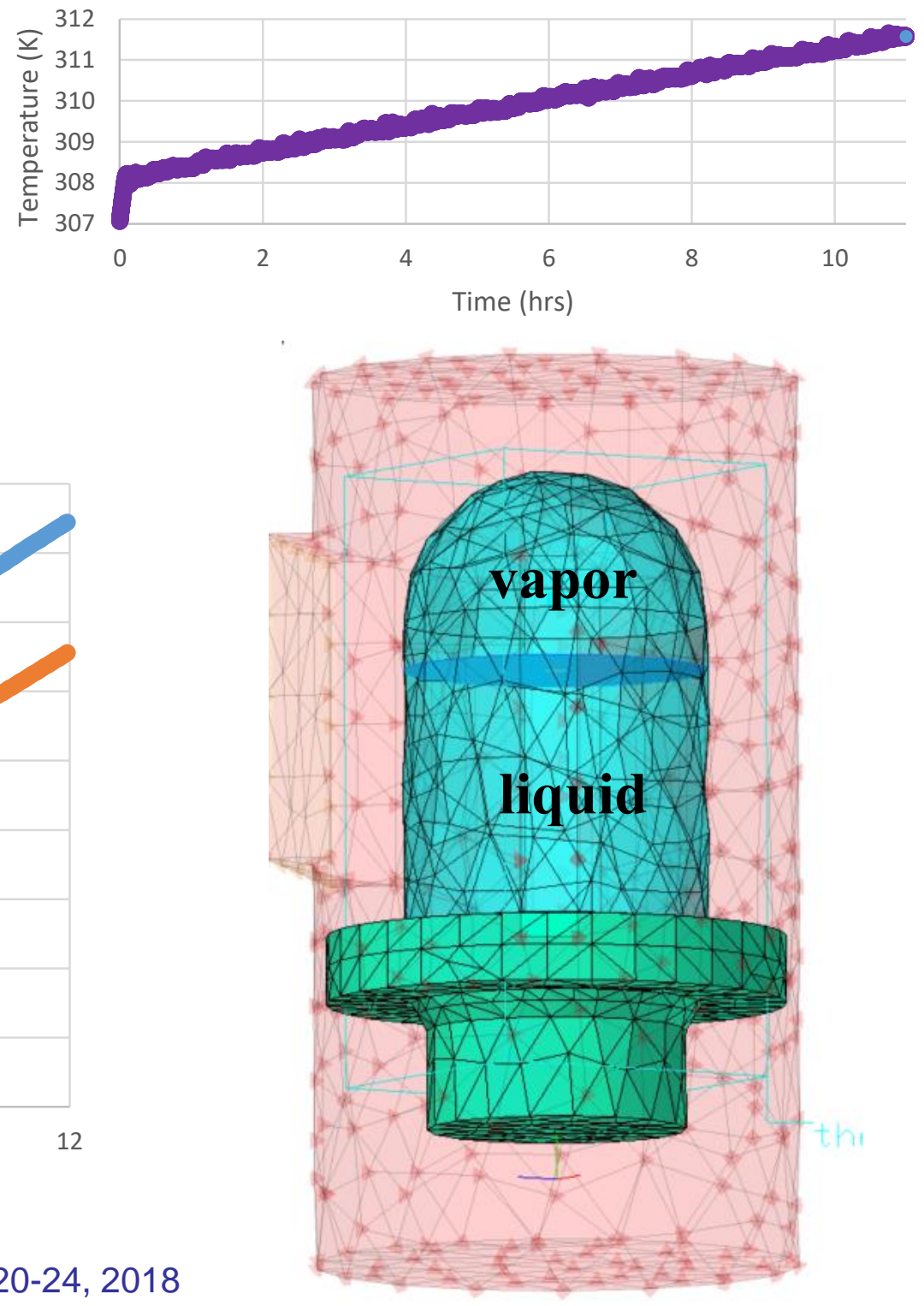
- Thermal Desktop and SINDA/FLUINT

- $\mathrm{Q}=0.5 \mathrm{~W}$

- Fill Level $=90 \%$

- Two Fluid Lumps

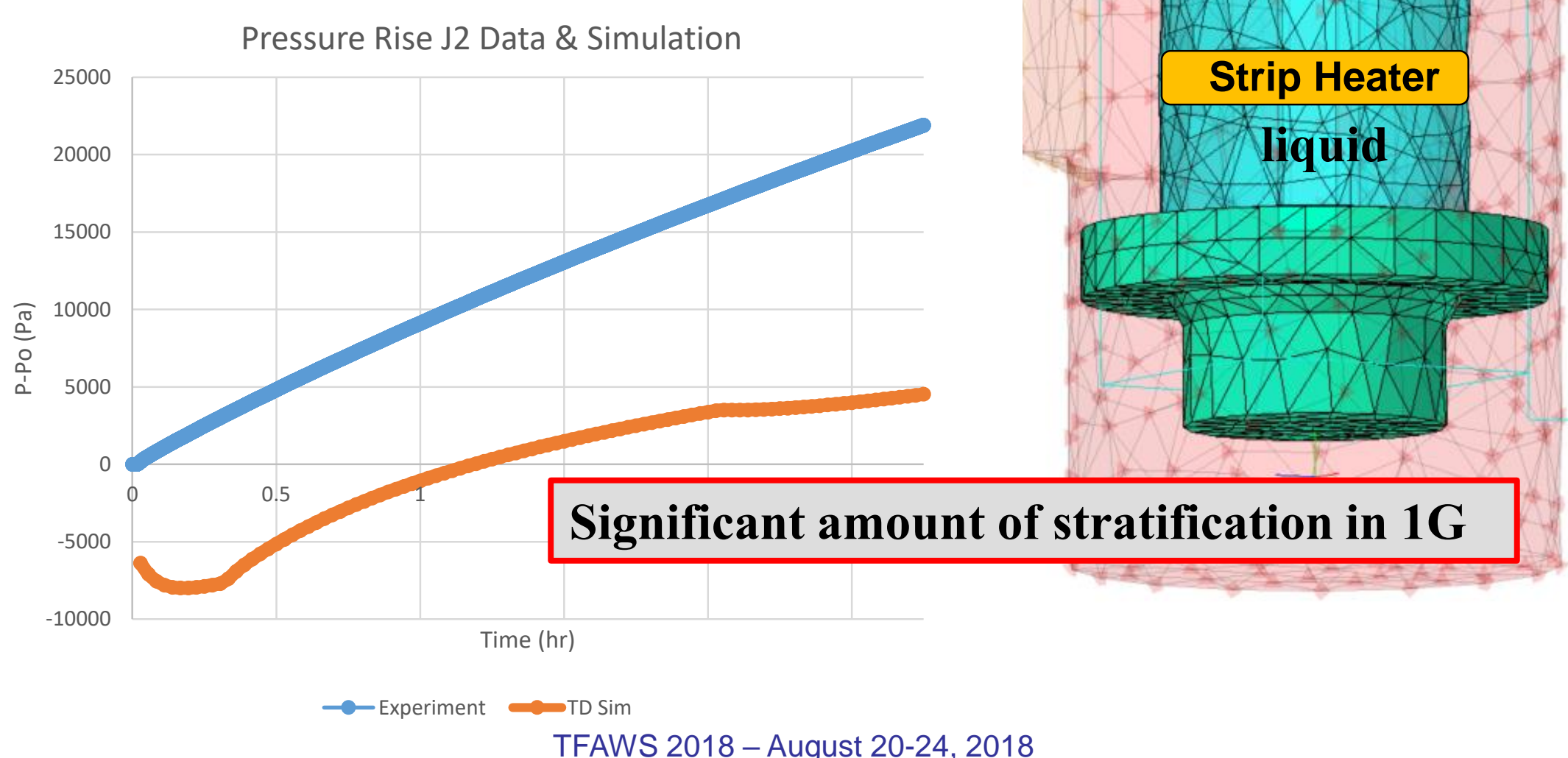


- Microgravity, Strip Heater case

- $\mathrm{Q}=0.5 \mathrm{~W}$

- Fill Level $=70 \%$

- Vapor/Liquid imported from initial Fluent 2D CFD model

- Liquid modeled as solid finite element

- 561 nodes

- Single fluid lump for vapor

- Heat and mass transfer between

Liquid/Vapor:

- Schrage Equation

$$
\begin{gathered}
|\dot{\mathbf{m}}|=\left(\frac{2 \sigma}{2-\sigma}\right)\left(\frac{M}{2 \pi R}\right)^{1 / 2}\left(\frac{P_{i}}{T_{i}^{1 / 2}}-\frac{P_{v}}{T_{v}^{1 / 2}}\right) \\
Q=\dot{m}_{\text {vap }} \\
\sigma=\underset{\text { accommodation coefficient }}{\text { TFAWS } 2018-\text { August } 20-24,2018}
\end{gathered}
$$

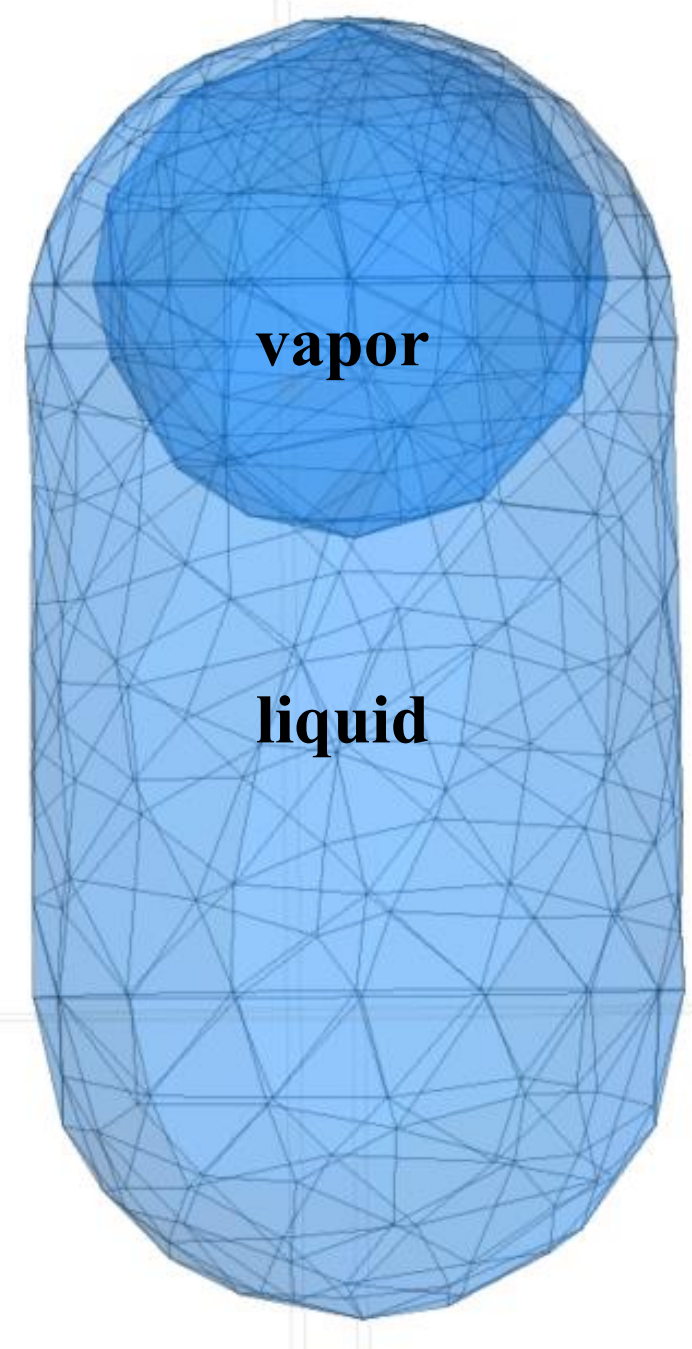


Model Validation: $\mu$ G Self-Pressurization, Strip Heater - No Mass Transfer NASA

Model Validation: $\mu$ Self-Pressurization, Strip Heater - No Mass Transfer
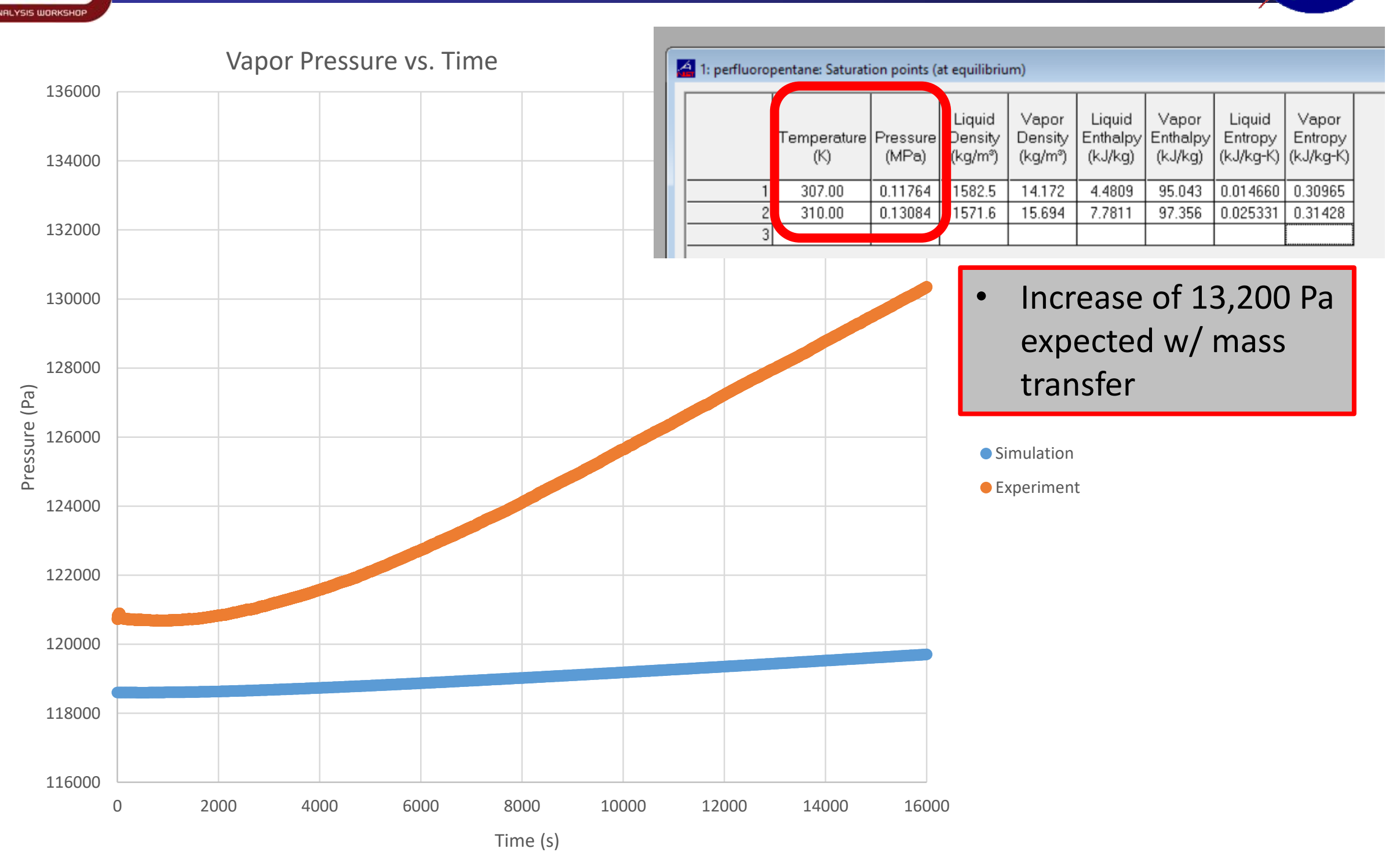

TFAWS 2018 - August 20-24, 2018 
Model Validation: $\mu$ G Self-Pressurization, Strip Heater, W/ Mass Transfer

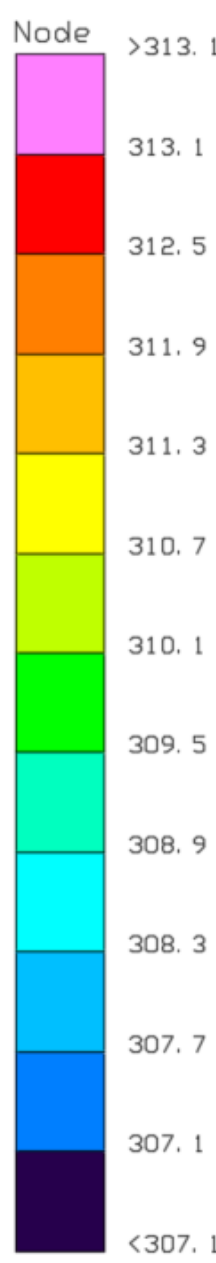

0.75 Hrs

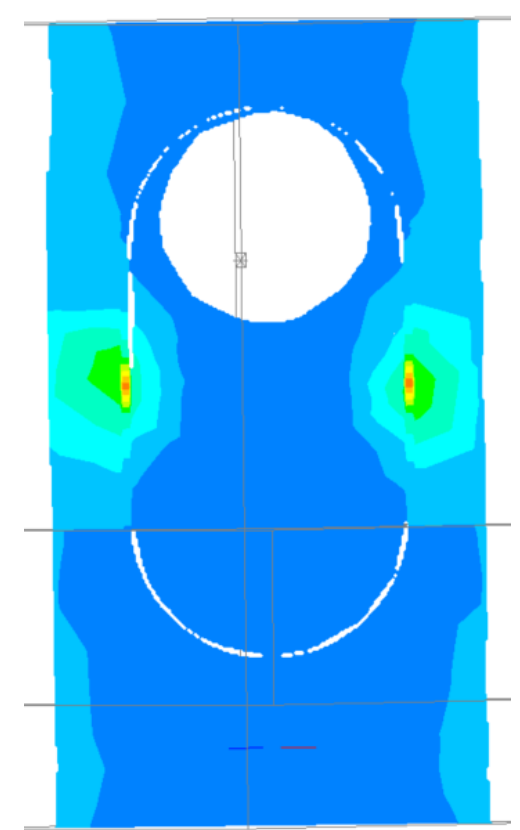

1.57 Hrs
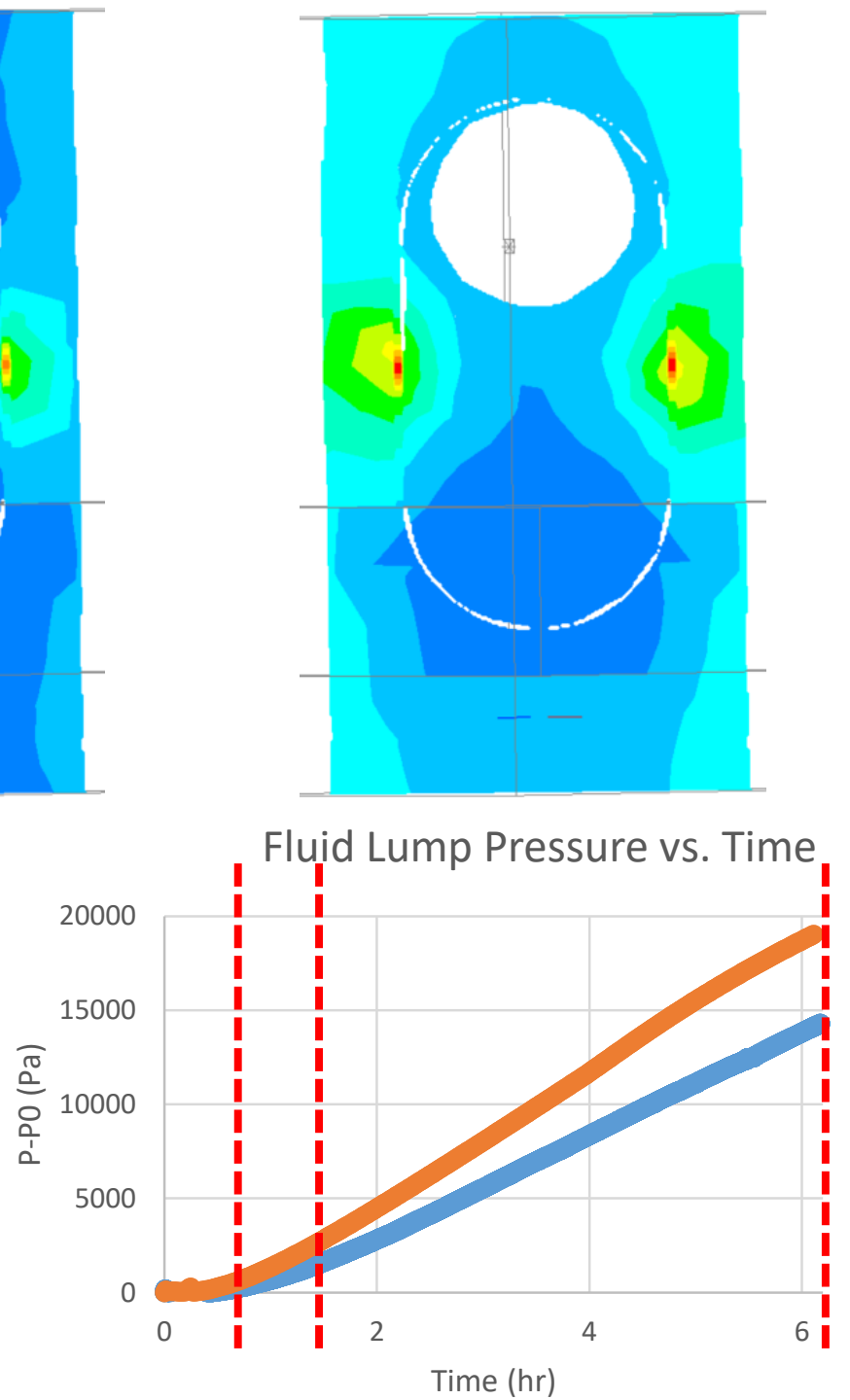

TFAWS 2018 - August 20-24, 2018

\subsection{Hrs}

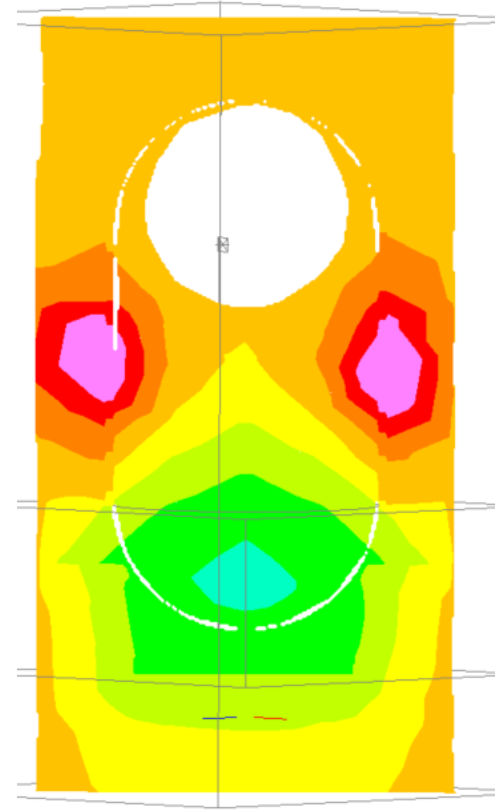

- Pres- 215 Data

- Pres- TDSim 
Model Validation: $\mu$ G Self-Pressurization, Strip Heater, W/ Mass Transfer

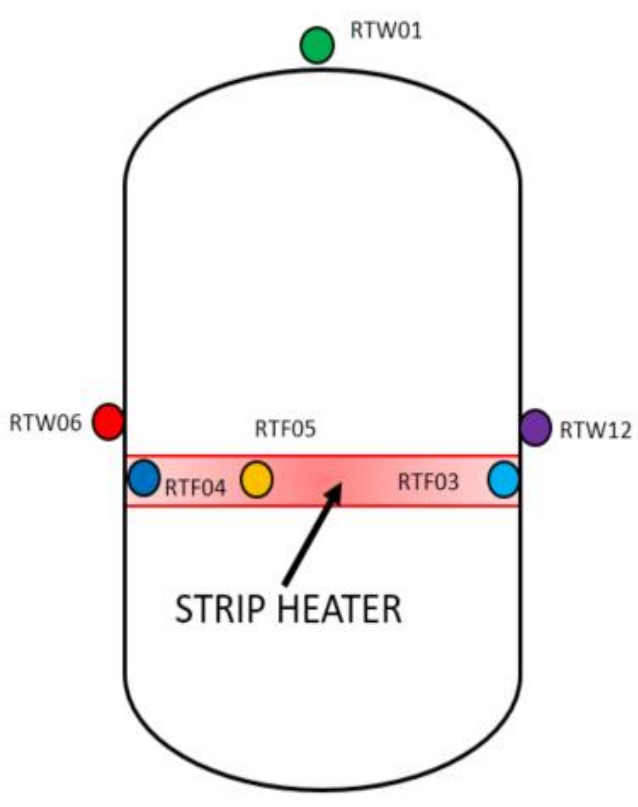

RTW01

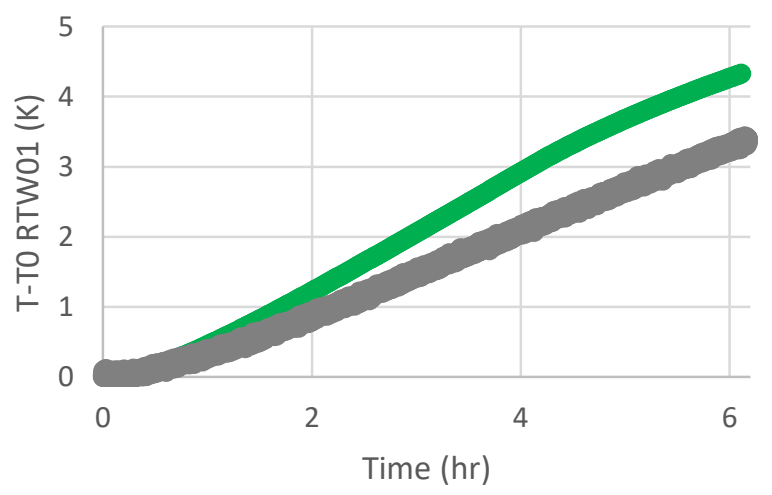

- RTW01,TDSim ORTW01,ExpData
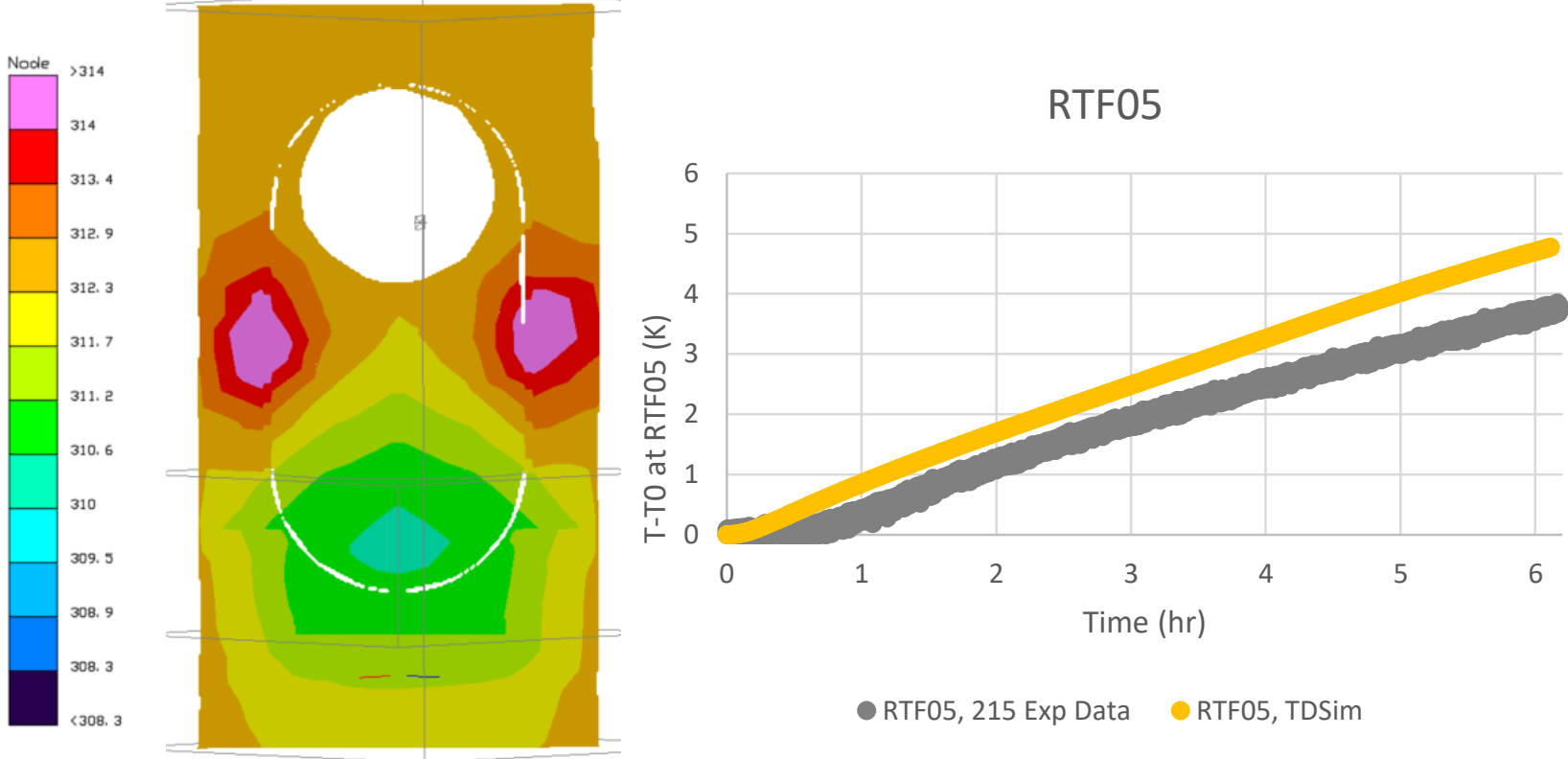

RTF05, 215 Exp Data RTF05, TDSim

RTF03, RTF04

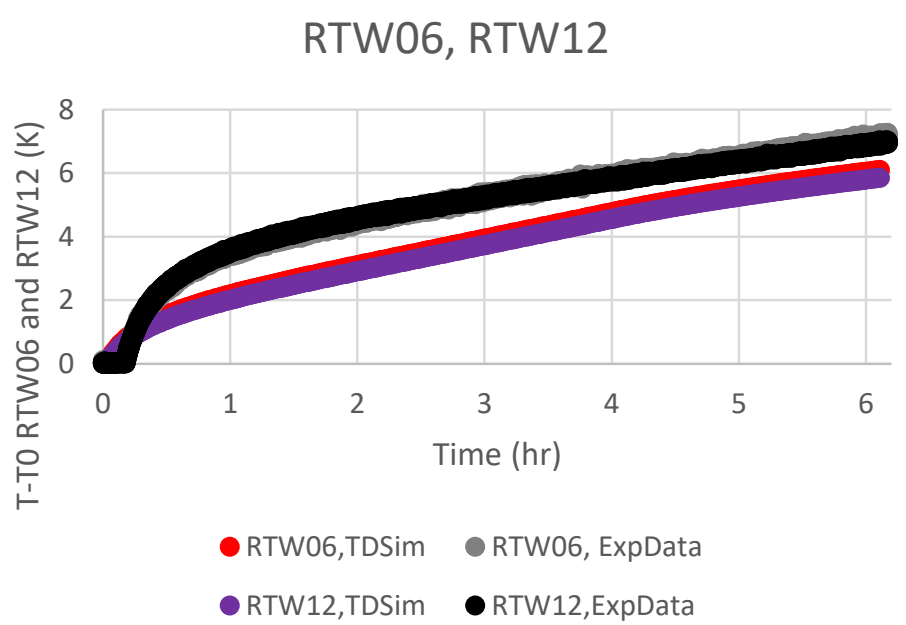

TFAWS 2018 - August 20-24, 2018

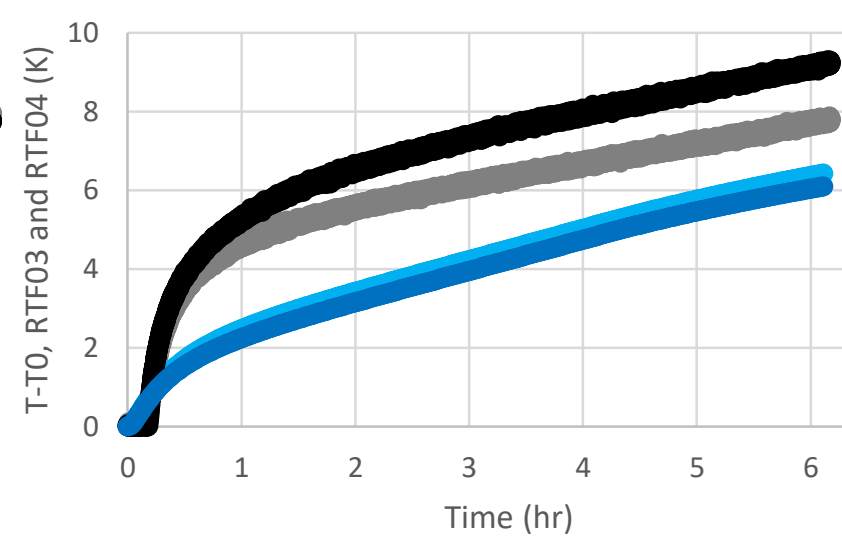

RTF03, 215 Exp Data RTF03,TDSim

- RTF04, 215 Exp Data RTF04,TDSim 12 


\section{Conclusions}

- Vacuum Jacket Heating Case, 1G:

- TD two-node fluid model able to match experimental pressure rise within $10 \%$

- Uniform heating of tank produces more uniform liquid temperatures within tank, causing more accurate results in model

- Strip Heater Case, 1G:

- TD two-node fluid model does poor job at matching experimental pressure rise due to localized heating of tank wall

- Strip Heater Case, $\mu \mathrm{G}$ :

- TD fluid model with finite element liquid able to match experimental pressure rise within 30\%, initial CFD results match experimental data within $10 \%$ 


\section{Future Work}

- Modeling of $1 \mathrm{G}$ case with Strip Heater

- Direct comparison with microgravity case

- Refine mesh of liquid finite element model

- Model won't run if accommodation coefficient is too large, CFD approach also had this problem

- CFD results using VOF can't resolve the grid at the LVI, have to use sharp interface

- Very fine grid near the LVI would allow wider range of accommodation coefficients

- Comparison of ZBOT results with cryogen in microgravity

- Further modeling efforts to focus on replication of larger tank in microgravity environment 


\section{Acknowledgements}

- Dr. Mohammed Kassemi (ZBOT PI, CWRU/GRC)

- Sonya Hylton (CWRU, GRC) 